Teil 1

Phänomenologie und Geschichte von »Fake News« und Desinformation 


\title{
Aktuelle Desinformation - Definition und Einordnung einer gesellschaftlichen Herausforderung
}

\author{
Fabian Zimmermann \& Matthias Kohring
}

Abstract

Unser Beitrag beschäftigt sich mit einer Form öffentlicher Irreführung, die gemeinhin unter "Fake News « firmiert. Zur präziseren Kennzeichnung dieses Phänomens verwenden wir den Begriff aktuelle Desinformation. Anhand expliziter Kriterien entwickeln wir eine Definition von aktueller Desinformation als Kommunikation wissentlich und empirisch falscher Informationen zu neuen und relevanten Sachverhalten mit dem Anspruch auf Wahrheit. Im Anschluss ordnen wir das Phänomen in den größeren sozialen Zusammenhang einer Desinformationsordnung ein und plädieren dafür, auf dieses gesellschaftliche Problem auch mit gesellschaftlichen Lösungen zu reagieren.

Noch bis vor Kurzem wurde unter »Fake News« ausschließlich politische Nachrichtensatire oder Nachrichtenparodie verstanden (Baym \& Jones, 2013), wie zum Beispiel die Daily Show in den USA, die heute-show mit Oliver Welke oder satirische Webseiten wie The Onion oder Der Postillon. »Fake News« als Nachrichtensatire wurde eine kritisch-aufklärerische Funktion zugeschrieben (Baym, 2005; Borden \& Tew, 2007; Reilly, 2012). Diese positive Sichtweise wird in der Literatur seit 2017 schlagartig durch ein negatives Verständnis von »Fake News« als im allgemeinsten Sinne (manipulative) Falschinformation abgelöst; als Auslöser für diesen Umschwung wird gemeinhin die US-amerikanische Präsidentschaftswahl von 2016 angesehen. Waren damit zu Anfang noch fingierte Informationsangebote im Internet gemeint, wurde der Ausdruck dann aber auch insbesondere von Politikern strategisch eingesetzt, um ihnen unliebsame Informationen gerade dieser etablierten Medien zu diskreditieren. Egelhofer und Lecheler (2019) unterscheiden daher zwischen »Fake News« als Genre und »Fake News« als Label. Ersteres beschreibt die vorsätzliche Herstellung pseudojournalistischer Falschinformationen, letzteres die politische Instrumentalisierung des Begriffs. Wir plädieren dafür, die Bezeichnung »Fake News« auf die strategische Etikettierung zu beschränken (Vosoughi, Roy \& Aral 
2018; Wardle \& Derakhshan, 2017) und nicht als wissenschaftlichen Begriff für eine spezifische Form der öffentlichen Irreführung weiterzuverwenden. Hierfür schlagen wir den Begriff der aktuellen Desinformation vor (Zimmermann \& Kohring, 2018).

Es besteht allerdings keineswegs ein Konsens, was genau unter aktueller Desinformation zu verstehen sei. Wenn man sich die zahlreichen Begriffsbestimmungen (in der Regel von »Fake News«) anschaut, stellt man fest, dass sie sehr unterschiedlich sind (Zimmermann \& Kohring, 2018). Weitgehend anerkannt sind nur zwei Definitionskriterien:

- Die Produktion von falschen oder potenziell irreführenden Informationen geschieht intentional.

- Diese Informationen werden als vermeintlich echte Nachrichten formatiert.

Bei weit mehr Kriterien ist dagegen keine Übereinkunft darüber ersichtlich, ob es sich um notwendige, d. h. unverzichtbare Kriterien für die Bestimmung von aktueller Desinformation handelt:

- Ist aktuelle Desinformation immer ein reines Online-Phänomen?

- Ist aktuelle Desinformation empirisch immer falsch?

- Ist aktuelle Desinformation immer mit einer Täuschungsabsicht verknüpft?

- Setzt die Bestimmung einer Nachricht als aktuelle Desinformation immer eine tatsächlich erfolgte Täuschung der Rezipienten voraus?

Zudem werden die bisher vorgelegten Definitionen nicht nachvollziehbar entwickelt, sondern fast immer einfach nur gesetzt, was eine direkte Vergleichbarkeit und Kritisierbarkeit verhindert. Ein solches Theoriedefizit hat ernstzunehmende Konsequenzen für das Verständnis des Phänomens aktueller Desinformation. Je nach Definition liegt der Fokus zum Beispiel nur auf empirisch falschen Tatsachenbehauptungen oder aber zusätzlich auf zum Beispiel Gerüchten, Verschwörungstheorien oder politischer Propaganda. Dies führt dazu, dass alle darüber reden und jeder etwas anderes meint - nicht zuletzt, wenn es darum geht, was man denn gegen aktuelle Desinformationen tun kann. 


\section{Definition aktueller Desinformation}

Die folgende Begriffsexplikation benennt die Kriterien, die unserer Ansicht nach für eine Bestimmung von aktueller Desinformation notwendig sind. Hierbei handelt es sich um die Kriterien (1) Kommunikation, (2) Aktualität, (3) Wahrheitsanspruch, (4) Unwahrheit und (5) Unwahrhaftigkeit. Das Kriterium (6) Täuschungsabsicht ist dagegen kein notwendiges Kriterium; mit seiner Hilfe lassen sich allerdings verschiedene Formen von aktueller Desinformation unterscheiden (Zimmermann \& Kohring, 2018; Kohring \& Zimmermann 2019).

\section{Kommunikation}

Desinformation richtet sich als Mitteilung an mindestens einen anderen sozialen Akteur und stellt mithin eine Form der Kommunikation dar. Der Desinformationsbegriff schließt dabei neben sprachlichen auch visuelle Mitteilungsformen ein (Fallis, 2014). Aktuelle Desinformation kann also auch in Form von (manipulierten) Fotografien und Videos (sogenannten Deep Fakes) oder sogenannten Memes Verbreitung finden. Nicht-kommunikative Formen der versuchten Irreführung wie die Geheimhaltung fallen indes nicht unter diesen Begriff. Die Mitteilung ist wiederum von der durch sie vermittelten Information, also ihrem Bedeutungsinhalt, zu unterscheiden. Die Notwendigkeit einer solchen Unterscheidung bemerkt man zum Beispiel bei ironischen oder satirischen Aussagen, die etwas ganz anderes (oft das Gegenteil) meinen, als sie vermeintlich mitteilen.

Eine Desinformation wird allerdings erst dann zum Bestandteil der sozialen Realität, wenn ein Adressat eine Mitteilung auch als intendierte Information erkannt hat. Das bedeutet allerdings nicht, dass Desinformation, um als solche bezeichnet werden zu können, auch erfolgreich sein, also geglaubt werden muss (Fallis, 2015). Der Status einer Mitteilung als Desinformation hängt also nicht zusätzlich von einem bestimmten Effekt beim Adressaten ab.

\section{Aktualität}

Das Besondere des hier behandelten Typs von Desinformation liegt im Aktualitätsbezug. Der Begriff der Aktualität ist hier nicht nur im zeitlichen Sinne zu verstehen. Vielmehr umfasst er neben dem Neuigkeitswert (zeitliche Komponente) von Informationen auch deren öffentliche Relevanz 
(soziale Komponente) (Merten, 1973; Scholl \& Weischenberg, 1998). Die spezifische Qualität von Journalismus besteht mit Blick auf Aktualität (insbesondere im Gegensatz zur Öffentlichkeitsarbeit) darin, aktuelle Fremdstatt Selbstdarstellungen anzubieten. Der etablierte Journalismus beobachtet die Gesellschaft und die Interdependenzen ihrer Teilbereiche wie Politik, Wirtschaft oder Recht also (idealiter) von einer unbeteiligten Warte aus (Kohring \& Hug, 1997).

Auch aktuelle Desinformation orientiert sich an diesen Aktualitätskriterien. Sie behandelt bevorzugt überraschende Themen mit potenziell großer Auswirkung auf die Gesellschaft. So ermöglicht sie ihrem Publikum die Ausbildung von (wenn auch empirisch unzutreffenden; siehe unten) Erwartungen und dadurch eine bestimmte Orientierung in einer komplexen Welt. Bei aktueller Desinformation handelt es sich somit um, wenn auch pervertierte, journalistische Kommunikation - nicht bloß um die Nachahmung journalistischer Darstellungsformen. Die mediale Form der Verbreitung ist für den Status aktueller Desinformation nicht maßgeblich: Zwar wird aktuelle Desinformation vornehmlich über das Internet und hier besonders über soziale Medien verbreitet, genauso wurde und wird sie aber auch offline verbreitet - nicht nur über Printmedien oder Rundfunk, sondern auch in der interpersonalen Kommunikation.

Abzugrenzen ist die aktuelle von nicht-aktueller Desinformation. Dazu gehören beispielsweise historische Lügen wie die Leugnung des Holocausts, die keine neue Information präsentieren, sondern schon bekannte Aussagen nur wiederholen. ${ }^{1}$ Zum anderen geht es bei aktueller Desinformation immer um öffentliche beziehungsweise journalistische Kommunikation. So gehört die bewusstseinsbezogene Lüge, bei der bloß über die eigenen Vor- oder Einstellungen getäuscht werden soll (Chisholm \& Feehan, 1977; Mahon, 2008; Thummes, 2013), nicht zum Gegenstandsbereich der aktuellen Desinformation. Diese bezieht sich als öffentliche Fremdbeobachtung stattdessen immer auf ein Ereignis beziehungsweise einen Sachverhalt in der gesellschaftlichen Umwelt des Urhebers (X desinformiert Y über Z; Kohring, 2006).

1 Natürlich können historischen Lügen mithilfe neuer vermeintlicher Belege wieder Neuigkeitswert und soziale Relevanz zugeschrieben werden. In diesem Fall hätte man es erneut mit aktueller Desinformation zu tun. 


\section{Wahrheitsanspruch}

Journalistische Nachrichten werden erst dann zu sozial verbindlichen Wirklichkeitsbeschreibungen, wenn sie sich nicht nur an Neuigkeitswert und Relevanz, sondern auch an Faktizität ausrichten (Scholl \& Weischenberg, 1998). Auch die aktuelle Desinformation vertritt diesen Wahrheitsanspruch. Erst diese (vorgetäuschte) Orientierung an Faktizität begründet ihren Status als (desinformierende) Nachricht.

Die Desinformation setzt also eine Situation voraus, in welcher der Sprecher die Wahrheit seiner Aussage gewährleistet (Carson, 2010). Da es sich um Kommunikation handelt, muss das Publikum diesen Wahrheitsanspruch aber auch als solchen wahrnehmen und akzeptieren (was es bei einer satirischen Aussage zum Beispiel nicht täte). Mithilfe des Kriteriums des Wahrheitsanspruches lässt sich die aktuelle Desinformation von Meinungsäußerungen (zum Beispiel dem journalistischen Kommentar, aber auch Hate Speech) abgrenzen. Stark tendenziöse und ideologisch gefärbte Berichterstattung (wie zum Beispiel von RT Deutsch) gehört demnach nicht zwangsläufig zum Spektrum der Desinformation, solange hier lediglich Werturteile gefällt werden (zum Beispiel »Merkel muss weg!«). Werturteile beziehungsweise normative Aussagen gehören nämlich nicht zur Klasse der Behauptungen und sind insofern auch nicht wahrheitsfähig. ${ }^{2}$ Auch bei der Nachrichtensatire handelt es sich zwar um aktuelle Kommunikation, jedoch nicht um Desinformation. Die einschlägige Quelle (zum Beispiel Der Postillon), bestimmte Stil- und Textmerkmale sowie Kontextinformationen kennzeichnen den (frei erfundenen) Inhalt als humoristische Kunstform und setzen den Wahrheitsanspruch als Kommunikationsnorm in der Rezeptionssituation in der Regel außer Kraft.

\section{Unwahrheit}

Im Gegensatz zu wahren Nachrichten löst aktuelle Desinformation den zuvor beschriebenen Wahrheitsanspruch nicht ein. Sie birgt stets die Gefahr, falsche Vorstellungen $\mathrm{zu}$ einem bestimmten Sachverhalt aufseiten des

2 Nach Albert (1971) ist ein Satz dann ein Werturteil, wenn er »1. den jeweils anvisierten Sachverhalt in positiver oder negativer Weise für das Verhalten (Stellungnahme oder Handeln) auszeichnet; 2. dabei ein normatives Prinzip (Wertstandard oder Verhaltensmaxime) als gültig unterstellt, das ein entsprechendes Verhalten fordert; und 3. eine präskriptive Erwartung involviert, daß die Adressaten des Satzes sich mit diesem Prinzip identifizieren und sich daher entsprechend verhalten« (214). 
Adressaten hervorzurufen (Fallis, 2015). Dieses irreführende Potenzial besitzen nur tatsächlich unzutreffende Behauptungen. Die aktuelle Desinformation ist also zwingend empirisch falsch (Hernon, 1995; Fallis, 2009; Floridi, 2011). ${ }^{3}$

Das Wahrheitsurteil bezieht sich dabei wie gesagt nicht auf die Mitteilung selbst (Satz, Bild, Meme etc.), sondern immer auf deren Bedeutungsinhalt (Fallis, 2009; Floridi, 2011; Habermas, 1973). Mithilfe dieser Differenzierung von Mitteilung und Information ist auch nachzuvollziehen, warum man mittels Halbwahrheiten, also falscher Implikaturen, desinformieren kann (Fallis, 2014). In diesem Fall impliziert die Mitteilung (zum Beispiel aufgrund des Kontexts) eine Bedeutung, die jedoch nicht direkt gesagt oder gezeigt wird. Wenn $\mathrm{Y}$, der X im Nebenzimmer versteckt hat, beispielsweise auf die Frage: »Wo ist X? «, antwortet: »Er ist um diese Zeit oft in der Bar um die Ecke« (was wahr ist), impliziert er, dass sich X jetzt gerade in der besagten Bar befindet (was falsch ist). Die Irreführung erfolgt bei solchen sogenannten Halbwahrheiten besonders geschickt, indem sich der Kommunikator den Unterschied zwischen Mitteilung und Information zunutze macht. Während die Mitteilung wörtlich genommen der Wahrheit entspricht, bleibt die Information selbst, also der eigentliche Bedeutungsinhalt, auch in diesem Spezialfall der sogenannten »true disinformation « (Fallis, 2015, 409) unwahr.

Die aktuelle Desinformation ist also immer eine Falschmeldung. Diese ist für ihr Publikum potenziell irreführend, da die berichteten Informationen nicht einer überprüfbaren sozialen Realität entsprechen. Anhand des Wahrheitskriteriums unterscheidet sich aktuelle Desinformation vom Gerücht, das (noch) unentschieden gegenüber der Unterscheidung wahr/falsch ist. Das Gerücht zeichnet sich insbesondere durch das Fehlen eines verbrieften Wahrheitsgehalts und mithin durch seine Unverbürgtheit aus. Die Geltung der mitgeteilten Informationen liegt hier allein im Bereich des Vorstellbaren (Fleck, 2014; Kirchmann, 2004). Zudem erweisen sich Gerüchte nicht selten als wahr.

\section{Unwahrhaftigkeit}

Die notwendige Bedingung der Unwahrheit reicht allerdings noch nicht aus, um den Begriff der aktuellen Desinformation trennscharf zu erfassen. Die 
Unwahrhaftigkeit kommt als entscheidendes Definitionskriterium hinzu. Damit ist gemeint, dass der Urheber selbst nicht an die Gültigkeit seiner Tatsachenbehauptung glaubt. Wie bei der Lüge (Mahon, 2015; Müller, 2007) so muss auch bei der Desinformation der Kommunikator davon überzeugt sein, dass die Information, die er mitteilt, nicht der Wahrheit entspricht (Fallis, 2009; Floridi, 2015). Das Desinformieren ist eine bewusste, unaufrichtige Handlung. Die Unwahrhaftigkeit markiert also die Trennlinie zwischen unwissentlicher Fehlinformation (misinformation) und vorsätzlicher Desinformation.

Die aktuelle Desinformation ist damit etwas grundlegend anderes als redaktionelle Fehler, die unbewusst, zum Beispiel aufgrund von Geldmangel oder Zeitdruck, entstehen. Auch wenn beide Arten Falschmeldungen und damit als potenziell irreführend einzustufen sind, unterscheiden sie sich hinsichtlich der Qualität der Falschinformation und deren moralischer Bewertung. Während man Produzenten fehlerhafter Nachrichten höchstens Fahrlässigkeit vorwerfen könnte (Stichwort: poor journalism), handeln die Urheber von aktueller Desinformation vorsätzlich.

Deutlich wird hierbei auch, dass der Begriff der sogenannten Verschwörungstheorie quer zu dem hier präsentierten Schema liegt. So werden Verschwörungstheorien häufig vom Urheber selbst aufrichtig geglaubt und sind daher nicht notwendigerweise unwahrhaftig (Fallis, 2015). Zudem können sie nicht nur unwahre, sondern genauso auch wahre Behauptungen umfassen - mehr noch: Verschwörungstheorien können sich insgesamt als zutreffend erweisen. Konstitutiv ist lediglich ihre inhaltliche Grundüberzeugung, »(wichtige) Ereignisse als Folge [...] geheimer Absprachen und Aktionen zu erklären « (Hepfer, 2015, 24). Verschwörungstheorien beziehungsweise einzelne Elemente davon können zwar unter Umständen in den Bereich aktueller Desinformation fallen, die Begriffe sind allerdings keinesfalls gleichzusetzen.

Übrigens wird keineswegs vorausgesetzt, dass aktuelle Desinformation direkt von einem unwahrhaftigen Kommunikator bezogen wird. Wissentlich in die Welt gesetzte Falschmeldungen können auch von Intermediären (Journalisten, Freunde etc.) weiterverbreitet werden, die irrtümlicherweise von der Wahrheit dieser Informationen ausgehen. Obwohl diese Intermediäre also lediglich unbewusst fehlinformieren, verlieren die Meldungen dennoch nicht ihren Status als Desinformation. 


\section{Täuschungsabsicht}

Zuletzt soll erörtert werden, ob aktuelle Desinformation notwendigerweise darauf abzielt, falsche Vorstellungen aufseiten des Rezipienten hervorzurufen, also mit einer Täuschungsabsicht verknüpft ist. Die Täuschungsabsicht verstehen wir in Anlehnung an Mahon (2008) als Intention, jemanden in den Glauben zu versetzen, ein behaupteter Sachverhalt sei wahr und/oder der Autor dieser Behauptung glaube den Sachverhalt selbst. Auch wenn im Kontext der Desinformation häufig auf eine Täuschungsabsicht seitens des Kommunikators verwiesen wird (Fetzer, 2004; Floridi, 2011; Hernon, 1995), schränkt diese definitorische Bedingung den Geltungsbereich der Desinformation zu stark ein. Beispielsweise werden Falschinformationen zum Teil nur deshalb in Wikipedia-Artikel eingebaut, um zu testen, ob und wie schnell diese von den Verantwortlichen korrigiert werden. Die Irreführung des Lesers wird hier nicht beabsichtigt. Dennoch ist diese sogenannte »side effect disinformation « (Fallis, 2014, 137-139) genau wie die vorsätzlich täuschende Desinformation intentionale Irreführung, da die unwahren Informationen wissentlich zu einem bestimmten Zweck verbreitet werden. Eine mögliche Irreführung des Publikums geschieht damit nicht zufällig, sondern wird bewusst in Kauf genommen.

Unsere Begriffsbestimmung umfasst also einerseits die propagandistische Desinformation, also intentional täuschende Falschmeldungen, deren Urheber dezidiert politische Ziele verfolgen (Jowett \& O'Donnell, 2012). Diese hegen die Absicht, die Vorstellungen der Rezipienten mittels unwahrer Behauptungen zu manipulieren, um deren Meinungen, Einstellungen und Handeln in eine bestimmte Richtung zu lenken. Andererseits gibt es aber auch wissentliche Falschmeldungen, die keine genuine Täuschungsabsicht verfolgen. Zum einen ist hier die Clickbait-Desinformation zu nennen, die sich falscher Informationen bedient, um Werbeeinnahmen im Internet zu generieren. Die reißerischen und teils absurden Überschriften solcher Artikel oder Videos sollen vor allem die Neugier der Rezipienten wecken und deren Aufmerksamkeit binden. Ob diese Falschmeldungen letztlich geglaubt werden oder nicht, ist für die Produzenten allerdings unerheblich. Ihr Zweck liegt lediglich in der Generierung höherer Klickzahlen zur ökonomischen Gewinnmaximierung. Schließlich kann aktuelle Desinformation auch, wie schon am Beispiel der Wikipedia-Artikel illustriert, eine erzieherische oder investigative Funktion übernehmen: Durch die gezielte Verbreitung von aufklärerischer Desinformation im Netz sollen Nutzer, und hierunter nicht zuletzt Journalisten, für das Risiko gezielter Falschmeldungen 
im Netz sensibilisiert und zu einer gründlicheren Prüfung von Informationen bewegt werden.

Aktuelle Desinformation kann somit definiert werden als Kommunikation wissentlich und empirisch falscher Informationen zu neuen und relevanten Sachverhalten mit dem Anspruch auf Wahrheit. Eine Täuschungsabsicht ist keine notwendige Bedingung.

\section{Aktuelle Desinformation und die Desinformationsordnung}

Aktuelle Desinformation bloß als einzelne wissentliche Unwahrheiten zu betrachten, greift aus einer gesellschaftstheoretischen Perspektive allerdings zu kurz. Man sollte das Problem vielmehr als systematischen Versuch der Destabilisierung demokratischer Institutionen und Prozesse verstehen. Aktuelle Desinformationen müssen demnach in einen breiteren sozialen Rahmen eingebettet werden. Einige Autoren sprechen in diesem Zusammenhang gar von einem postfaktischen Zeitalter, in dem die vormals vertrauten Mechanismen der Wissensproduktion fundamental infrage gestellt werden (Gibson, 2018; Harsin, 2015): Ein namhafter Anteil sozialer Akteure hält sich nicht (mehr) an die konventionellen Kriterien der Faktizität wie Recherche, Beweisführung und Widerspruchsfreiheit und verlangt nach einer alternativen Weltsicht (Lewandowsky, Ecker \& Cook, 2017). Der Grund hierfür ist das zunehmende Misstrauen gegenüber den demokratischen Institutionen (Wissenschaft, Politik, klassische Nachrichtenmedien etc.) bei einem Teil der Bevölkerung.

Die Urheber aktueller Desinformation machen sich genau dieses Vertrauensdefizit zunutze und liefern einen Gegenentwurf zur gegenwärtigen demokratischen Ordnung. Sie wirken den Wirklichkeitsbeschreibungen der etablierten Institutionen entgegen und versorgen ihre Anhänger systematisch mit sogenannten Alternativen Fakten. Miteinander verknüpft ergeben sich daraus Narrative, also emotional und ideologisch aufgeladene Erzählungen, die eine bestimmte Sicht auf die Welt verkörpern und Orientierung stiften. Die Gesellschaft sieht sich also nicht bloß einzelnen Falschinformationen gegenüber, sondern einer umfassenden Desinformationsordnung. Dieses System von Desinformationen speist sich in den meisten Ländern aus rechtem Gedankengut (Bennett \& Livingston, 2018). Es zeichnet das Bild einer gescheiterten Demokratie mit korrupten Politikern, lügenden Journalisten und kriminellen Ausländern. In Deutschland steht vor allem die Ausgrenzung und Diffamierung (muslimischer) Migranten im Vorder- 
grund, da die Geflüchtetensituation seit 2015 lange die Schlagzeilen beherrschte (Humprecht, 2019).

\section{Verbreitung und Konsequenzen aktueller Desinformation}

Die Distribution aktueller Desinformation erfolgt vorrangig über digitale, insbesondere über soziale Medien wie Facebook oder Twitter. Diese ermöglichen prinzipiell jedem Akteur, Informationen zu aktuellen Themen und Ereignissen kostengünstig an potenziell unbegrenzte Publika zu verbreiten, was auch die massenhafte Veröffentlichung wissentlich falscher Tatsachenbehauptungen erleichtert. So kommt der größte Anteil der Zugriffe auf Desinformationswebsites nicht durch einen direkten Browseraufruf, sondern durch Links von Social-Media-Seiten zustande (Allcott $\&$ Gentzkow, 2017). Desinformationen verbreiten sich in sozialen Medien wesentlich schneller als wahre Nachrichten und tauchen in abgewandelter Form immer wieder auf (Shin, Jian, Driscoll \& Bar, 2018; Vosoughi, Roy \& Aral, 2018).

Vor diesem Hintergrund könnte aktuelle Online-Desinformation als ein Phänomen mit enormer Reichweite und umfassenden gesellschaftlichen Auswirkungen erscheinen. Dass diese Befürchtung, die nicht zuletzt durch die mediale Berichterstattung über »Fake News « genährt wurde, wohl überzogen sein dürfte, zeigen mehrere Studien. So macht Desinformation im Durchschnitt mit etwa fünf Prozent nur einen geringen Teil des gesamten Medienkonsums eines Rezipienten aus (Allcott \& Gentzkow, 2017; Grinberg, Joseph, Friedland, Swire-Thompson \& Lazar, 2019). Man kann also zum jetzigen Zeitpunkt nur schwerlich davon ausgehen, dass aktuelle Desinformation in der Lage ist, die Masse an wahren Nachrichten aus der Medienlandschaft zu verdrängen und die Gesellschaft als Ganzes zu beeinflussen.

Allerdings täuscht diese Aggregierung über alle Mediennutzer darüber hinweg, dass die Rezeption von Desinformationen im Netz extrem konzentriert ist und es sich somit um einen Nischenbereich handelt. Das Publikum aktueller Desinformation besteht aus einer relativ kleinen Bevölkerungsgruppe mit spezifischen Eigenschaften (Nelson \& Taneja, 2018). Insbesondere Konservative und Ältere sind hierfür anfällig. Im Gegensatz zum Rest kommt dieser Teil der Gesellschaft sehr häufig mit Desinformationen in Kontakt (Grinberg et al., 2019; Hjorth \& Adler-Nissen, 2019). Die Theorie des selective exposure legt nahe, dass sich diese Personen bewusst sogenannten Alternativen Fakten zuwenden, da sie ihre (zumeist rechten) 
politischen Ansichten bekräftigen (Guess, Nyhan \& Reifler, 2018). Mit Blick auf diese Gruppe zeigt sich also doch ein Einfallstor für Effekte aktueller Desinformation.

Die Forschungsliteratur weist auf mehrere mögliche Konsequenzen von Falschinformationen und Fehlwahrnehmungen hin. So führt die Verbreitung von Desinformationen über politische Akteure oder Sachthemen (zum Beispiel Sozialhilfe, Arbeitslosigkeit, Umwelt) zu falschen Vorstellungen, die die Präferenz für bestimmte politische Kandidaten und Maßnahmen prägen können (Kuklinski, Quirk, Jerit, Schwieder \& Rich, 2000; Kull, Ramsay \& Lewis, 2003; Thorson, 2016). Auch tiefer liegende politische Einstellungen unterliegen der Beeinflussung durch Desinformation: Huang (2017) zeigt, dass negative Falschbehauptungen über die Regierung auf sozialen Medien das politische Vertrauen und die politische Unterstützung der Bürger vermindern.

Außerdem gibt es empirische Belege für den Einfluss von politischer Desinformation auf das Herzstück der Demokratie - die Wahlentscheidung. Mehrere Untersuchungen deuten darauf hin, dass manipulierte Vorstellungen über politische Akteure und Sachverhalte das Abstimmungsverhalten der Bürger beeinflussen - zusätzlich zum Einfluss ihrer allgemeinen politischen Einstellungen (Reedy, Wells \& Gastil, 2014; Wells, Reedy, Gastil \& Lee, 2009; Weeks \& Garrett, 2014). Auch das Ergebnis des britischen Referendums zum Verlassen der Europäischen Union (Brexit) ist wohl zum Teil auf falsche, islamophobe Verschwörungstheorien zurückzuführen (Swami, Barron, Weis \& Furnham, 2018). Ähnliches gilt auch für die Präsidentschaftswahlen in Frankreich und den USA. Barrera, Guriev, Henry und Zhuravskaya (2018) belegen, dass die Rezeption irreführender Behauptungen zur europäischen Geflüchtetensituation die Wahlchancen von Marine Le Pen erhöht hat. Ebenso trugen gegen Hillary Clinton gerichtete Desinformationen 2016 offenbar dazu bei, dass ehemalige Obama-Wähler zu Donald Trump überliefen oder ins Lager der Nichtwähler wechselten (Gunther, Beck \& Nisbet, 2019). Auch im Kontext der Wahl zum Deutschen Bundestag im Jahr 2017 gibt es empirische Belege für einen spezifischen Einfluss auf die Wahlentscheidung. Das Glauben politischer Desinformation entfremdete insbesondere konservative Wähler von der regierenden CDU/CSU und veranlasste sie stattdessen zur Wahl der populistischen AfD (Zimmermann \& Kohring, 2020).

Die gerade skizzierten Konsequenzen aktueller Desinformation ergeben sich nicht primär aus ihrem (fehlenden) Wahrheitsgehalt oder ihrer (fehlenden) Wahrhaftigkeit (die im Falle einer erfolgreichen Irreführung ja verkannt werden). Sie hängen vielmehr von den implizierten Narrativen ab, die 
durch die meist rechtspopulistisch gefärbten Falschinformationen vermittelt werden.

\section{Maßnahmen gegen aktuelle Desinformation}

Angesichts ihres Potenzials, die Grundlagen politischer Willensbildung zu beeinflussen, erscheinen Maßnahmen gegen aktuelle Desinformation durchaus geboten. Diese können sich prinzipiell auf drei Aspekte des Kommunikationsprozesses richten: (a) die Produktion und Distribution, (b) den Inhalt und (c) die Nutzung und Rezeption.

\section{Produktion und Distribution aktueller Desinformation}

In Deutschland gelten für die Produzenten von Internetangeboten (sogenannten Telemedien), die nicht Rundfunk sind, noch nicht einmal die Mindeststandards an Ausgewogenheit und Pluralität, die im 4. Rundfunkurteil von 1986 des Bundesverfassungsgerichts für die privaten Rundfunkveranstalter festgelegt wurden. Urheber unwahrer Tatsachenbehauptungen sind daher nicht schon deshalb sanktionierbar, weil sie diese verbreiten. Als Tatsachenbehauptung ist in Deutschland nur die Holocaust-Leugnung verboten; ansonsten müssen Straftatbestände wie Volksverhetzung, Verletzung von Persönlichkeitsrechten oder Verbreitung von Propaganda einer verfassungswidrigen Organisation erfüllt sein. Selbst unter der Annahme, dies sei in vielen Fällen propagandistischer Desinformation gegeben (was nicht zwingend der Fall sein muss), gestaltet sich die Strafverfolgung der tatsächlichen Urheber äußerst schwierig. Nicht selten stammt aktuelle Desinformation von anonymen Quellen aus dem Ausland (zum Beispiel aus einer russischen Trollfabrik) und verbreitet sich über soziale Medien weiter. Neben der mangelnden Identifizierbarkeit erschwert also auch die eingeschränkte rechtliche Zuständigkeit Maßnahmen auf der Produktionsseite.

Deshalb wird verstärkt darauf gesetzt, die Distributoren von Desinformation in die Pflicht zu nehmen. So verpflichtet das deutsche Netzwerkdurchsetzungsgesetz von 2017 die großen Social-Media-Plattformen, nach Beschwerden rechtswidrige Inhalte innerhalb relativ kurzer Zeit zu entfernen oder zu sperren (im Prinzip sah dies auch schon das Telemediengesetz von 2007 vor). Die sozialen Netzwerke sind zwar weiterhin nicht verpflichtet, von sich aus nach rechtswidrigen Inhalten zu suchen - sie könnten aber nun darauf bedacht sein, vorsorglich bedenkliche Inhalte (automatisiert) zu löschen und somit Beschwerden und kostspielige Sanktionen zu vermeiden. 
Solche algorithmenbasierten Mechanismen (zum Beispiel Upload-Filter) könnten allerdings auch der Löschung rechtlich unbedenklicher Inhalte Vorschub leisten. Darüber hinaus überlässt man so die Entscheidung zur Löschung den Betreibern, nicht Gerichten. Auch ein Vorgehen gegen Desinformation verbreitende Social Bots - automatisierte Accounts, hinter denen keine realen Menschen, sondern eine Programmierung steckt - wird diskutiert. Allerdings variieren unterschiedliche Bot-Detektoren (zum Beispiel Botometer, Botcheck etc.) dahingehend, welche und wie viele Accounts sie (mit einer gewissen Wahrscheinlichkeit) als Social Bots ausweisen. Zudem entwickeln sich Bots sehr schnell weiter, sodass automatisierte Erkennungsmethoden nur eine kurze Halbwertszeit haben und ständiger Überarbeitung bedürfen. Die zuverlässige und zweifelsfreie Identifikation politisch schädlicher Bots ist also zumindest momentan noch fragwürdig. Auch überschätzt dieser Ansatz wohl die Rolle von Social Bots für die Distribution von Desinformation. Deren Verbreitung ist nämlich mehr auf die Aktivität von realen Menschen als auf die von programmierten Robotern zurückzuführen (Vosoughi, Roy \& Aral, 2018).

\section{Inhalt potenzieller Desinformationen}

Auf der Inhaltsseite gibt es grundsätzlich zwei Möglichkeiten, mit aktueller Desinformation umzugehen: (A) Faktencheck: Eine Information wird überprüft und gegebenenfalls mit einem Hinweis beziehungsweise einem Link zur Korrektur versehen; (B) Löschung: Ein als Desinformation identifizierter Inhalt wird gesperrt oder gelöscht.

(A) Der Faktencheck konfrontiert unwahre Tatsachenbehauptungen mit einer eigens recherchierten Gegendarstellung. Da dies sehr aufwändig ist, werden auch automatisierte Prozeduren des Machine Learnings eingesetzt, die den menschlichen Faktenchecker aber (noch) nicht ersetzen können (Graves, 2018). Die Skepsis gegenüber der Wirksamkeit von Faktenchecks liegt aber auf einer anderen Ebene: Zum Ersten erreichen sie diejenigen Publika, die aktuelle Desinformation rezipieren und glauben, nur selten (Nyhan et al., 2019). Zum Zweiten werden sie selbst im Falle einer Rezeption von diesen Publika in der Regel eher abgelehnt: Faktenchecks kommen oft von Journalisten oder den Nachrichtenmedien selbst und werden von diesen überaus skeptischen Publika als nicht unparteiisch und daher nicht vertrauenswürdig erachtet. Zum Dritten ist selbst dann, wenn ein Rezipient eine einzelne Faktenkorrektur rezipiert und akzeptiert, die Wirkung auf politische Einstellungen gering (Nyhan et al., 2019; Barrera et al., 2018). 
Aktuelle Desinformationen sind nämlich in bestimmte Narrative eingebettet und von diesen nicht zu isolieren (Ecker \& Ang, 2019). Die durch die Falschmeldung wachgerufene abstrakte Erzählung (zum Beispiel über kriminelle Ausländer) wird durch die Korrektur konkreter Behauptungen nicht ernsthaft erschüttert.

(B) Das möglichst schnelle Löschen aktueller Desinformation wäre daher effektiver, da es wenigstens den weiteren Kontakt mit ihr verhinderte. Die rechtmäßige Löschung einer öffentlichen Äußerung im Netz setzt aber natürlich die Rechtswidrigkeit der betreffenden (Des-)Information voraus (siehe oben). Zusätzliche Gesetze, die Falschmeldungen als solche verbieten, sind prinzipiell möglich; sie gibt es zum Beispiel in einigen asiatischen Staaten und seit Ende Dezember 2018 auch in Frankreich. Hier sieht das Gesetz vor, dass in den drei Monaten vor einer landesweiten Wahl die Verbreitung von politischen Falschinformationen durch richterliche Eilbeschlüsse unterbunden werden kann.

Prinzipiell könnten auch automatisierte Verfahren zur Erkennung und Löschung aktueller Desinformation eingesetzt werden. Diese Verfahren müssten aber nicht nur falsche Informationen einwandfrei identifizieren, sondern auch deren Rechtswidrigkeit (siehe oben) zweifelsfrei feststellen. In beiden Fällen würde es immer auch zu Fehleinschätzungen kommen, das heißt, es würden auch wahre oder bloß ungesicherte Informationen mitgelöscht. Schon die bloße Existenz einer solchen Kontrolle würde allerdings Befürchtungen eines möglichen Missbrauchs schüren und gesellschaftliches Misstrauen gegenüber kommerziellen und/oder staatlichen Institutionen befördern.

\section{Nutzung und Rezeption aktueller Desinformation}

Insgesamt betrachtet ist die Bekämpfung von Desinformation auf der Produktions-, Distributions- und Inhaltsseite mit rechtlichen, verfahrenstechnischen und nicht zuletzt demokratietheoretischen Problemen behaftet. Daher lohnt es sich, nach Maßnahmen im Bereich Nutzung und Rezeption zu suchen. Hierfür sollte man zunächst die Annahme aufgeben, dass Nutzer beziehungsweise Rezipienten als passive Opfer von Desinformationen anzusehen seien. (Die Diskussion trägt ja durchaus einen bewahrpädagogischen Charakter). Rezipienten wenden sich alternativen Informationsquellen - darunter eben auch aktueller Desinformation - meist aktiv zu, um ihre Einstellungen zu bestätigen oder um eine den etablierten Institutionen gegenüber konträre Weltsicht zu erhalten. Dabei geht es weniger um die einzelnen 
(Des-)Informationen, sondern um die ideologischen Narrative, in die diese eingebettet sind.

Daher ist es unzureichend, aktueller Desinformation bloß Richtigstellungen entgegenzusetzen. Auch Überlegungen zu einer automatisierten Erkennung und Löschung reagieren auf ein gesellschaftliches Problem nur mit technischen Lösungen. Maßnahmen, um die Verbreitung und den Einfluss von Desinformation zurückzudrängen, müssten aber vor allem das Soziale in den Blick nehmen. Dazu zählen die Vermittlung politischer Bildung und Programme gegen rechte Ideologie genauso wie die Förderung einer kritischen Medienkompetenz. Vor allem aber gilt es, das verlorene Institutionenvertrauen wiederzugewinnen. Hier geht es darum nachzuvollziehen, warum ein gewisser Teil der Bevölkerung vor allem Politik und Medien das Vertrauen weitgehend entzogen hat (in Deutschland sind das mindestens 20 Prozent der Bevölkerung; Bayerischer Rundfunk, 2016; Jackob, Schultz, Ziegele, Quiring \& Schemer, 2019) und deshalb vermehrt nach Alternativen sucht. Dafür müssen sich Politik und Medien auch auf die Debatte einlassen, inwiefern die Gründe des Vertrauensverlusts mitverantwortet sind und ob sie mehr als bisher tun müssten, um Vertrauen zurückzugewinnen.

\section{Literatur}

Albert, Hans (1971). Theorie und Praxis. Max Weber und das Problem der Wertfreiheit und der Rationalität. In: Albert, H. \& E. Topitsch (Hrsg.), Werturteilsstreit (200-236). Darmstadt: Wissenschaftliche Buchgesellschaft.

Allcott, Hunt \& Gentzkow, Matthew (2017). Social Media and Fake News in the 2016 Election. Journal of Economic Perspectives, 31, 211-235. DOI: 10.1257/jep.31.2. 211.

Barrera, Oscar; Guriev, Sergei M.; Henry, Emeric \& Zhuravskaya, Ekaterina (2018). Facts, Alternative Facts, and Fact Checking in Times of Post-Truth Politics. Online Vorveröffentlichung. DOI: 10.2139/ssrn.3004631.

Bayerischer Rundfunk (2016). Informationen fürs Leben. BR-Studie zum Vertrauen in die Medien. München.

Baym, Geoffrey (2005). The Daily Show. Discursive Integration and the Reinvention of Political Journalism. Political Communication, 22, 259-276. DOI: 10.1080/ 10584600591006492.

Baym, Geoffrey \& Jones, Jeffrey P. (Hrsg.) (2013). News Parody and Political Satire Across the Globe. London: Routledge.

Bennett, W. Lance \& Livingston, Steven (2018). The Disinformation Order. Disruptive Communication and the Decline of Democratic Institutions. European Journal of Communication, 33, 122-139. DOI: 10.1177/0267323118760317. 
Borden, Sandra L. \& Tew, Chad (2007). The Role of Journalist and the Performance of Journalism. Ethical Lessons from »Fake« News (Seriously). Journal of Mass Media Ethics, 22, 300-314. DOI: 10.1080/08900520701583586.

Carson, Thomas L. (2010). Lying and deception. Theory and practice. New York, NY: Oxford University Press.

Chisholm, Roderick M. \& Feehan, Thomas D. (1977). The Intent to Deceive. The Journal of Philosophy, 74, 143-159. DOI: 10.2307/2025605.

Ecker, Ullrich K. H. \& Ang, Li Chang (2019). Political Attitudes and the Processing of Misinformation Corrections. Political Psychology, 40, 241-260. DOI: 10.1111/ pops. 12494.

Egelhofer, Jana Laura \& Lecheler, Sophie (2019). Fake News as a Two-Dimensional Phenomenon. A Framework and Research Agenda. Annals of the International Communication Association, 43(2), 97-116. DOI: 10.1080/23808985.2019.1602782.

Fallis, Don (2009). A Conceptual Analysis of Disinformation. iConference 2009 Proceedings. Abgerufen von http://hdl.handle.net/2142/15205.

Fallis, Don (2014). The Varieties of Disinformation. In: Floridi, L. \& P. Illari (Hrsg.), The Philosophy of Information Quality (135-161). Cham: Springer.

Fallis, Don (2015). What is Disinformation? Liberary Trends, 63, 401-426. DOI: 10.1353/lib.2015.0014.

Fetzer, James H. (2004). Disinformation. The Use of False Information. Minds and Machines, 14, 231-240. DOI: 10.1023/B:MIND.0000021683.28604.5b.

Fleck, Jan (2014). Das Gerücht als Kommunikation im Massenmedium WWW. Überlegungen zu Beobachtbarkeit und theoretischer Kontextualisierung. In: Malsch, T. \& M. Schmitt (Hrsg.), Neue Impulse für die soziologische Kommunikationstheorie. Empirische Widerstände und theoretische Verknüpfungen (187-213). Wiesbaden: Springer VS.

Floridi, Luciano (2011). The Philosophy of Information. New York, NY: Oxford University Press.

Floridi, Luciano (2015). Semantic Conceptions of Information. The Stanford Encyclopedia of Philosophy (Spring 2017 Edition). Abgerufen von https://plato. stanford.edu/archives/spr2017/entries/information-semantic. Abgerufen am 19. September 2019.

Gibson, Timothy A. (2018). The Post-Truth Double Helix. Reflexivity and Mistrust in Local Politics. International Journal of Communication, 12, 3167-3185.

Graves, Lucas (2018). Understanding the Promise and Limits of Automated Fact-Checking. Oxford, UK: Reuters Institute for the Study of Journalism.

Grinberg, Nir; Joseph, Kenneth; Friedland, Lisa; Swire-Thompson, Briony \& Lazar, David (2019). Fake News on Twitter During the 2016 U.S. Presidential Election. Science, 363, 374-378.

Guess, Andrew; Nyhan, Brendan \& Reifler, Jason (2018). Selective Exposure to Misinformation. Evidence From the Consumption of Fake News During the 2016 U.S. Presidential Campaign. European Research Council. Abgerufen von https://about.fb.com/wp-content/uploads/2018/01/fake-news-2016.pdf. 
Gunther, Richard; Beck, Paul A. \& Nisbet, Erik C. (2019). »Fake News« and the Defection of 2012 Obama Voters in the 2016 Presidential Election. Electoral Studies, 42. Online Vorveröffentlichung. DOI: 10.1016/j.electstud.2019.03.006.

Habermas, Jürgen (1973). Wahrheitstheorien. In: Fahrenbach, H. (Hrsg.), Wirklichkeit und Reflexion. Walter Schulz zum 60. Geburtstag (211-266). Pfullingen: Neske.

Harsin, Jayson (2015). Regimes of Posttruth, Postpolitics, and Attention Economies. Communication, Culture \& Critique, 8, 327-333. DOI: 10.1111/cccr.12097.

Hepfer, Karl (2015). Verschwörungstheorien. Eine philosophische Kritik der Unvernunft. Bielefeld: Transcript.

Hernon, Peter (1995). Disinformation and Misinformation Through the Internet. Findings of an Exploratory Study. Government Information Quarterly, 12, 133-139. DOI: 10.1016/0740-624X(95)90052-7.

Hjorth, Frederik \& Adler-Nissen, Rebecca (2019). Ideological Asymmetry in the Reach of Pro-Russian Digital Disinformation to United States Audiences. Journal of Communication, 69, 168-192. DOI: 10.1093/joc/jqz006.

Huang, Heifeng (2017). A War of (Mis)information. The Political Effects of Rumors and Rumor Rebuttals in an Authoritarian Country. British Journal of Political Science, 47, 283-311. DOI: 10.1017/S0007123415000253.

Humprecht, Edda (2019). Where »Fake News« Flourishes. A Comparison Across Four Western Democracies. Information, Communication \& Society, 22, 1973-1988. DOI: 10.1080/1369118X.2018.1474241.

Jackob, Nikolaus; Schultz, Tanjev; Ziegele, Marc; Quiring, Oliver \& Schemer, Christian (2019). Medienvertrauen im Zeitalter der Polarisierung. Mainzer Langzeitstudie Medienvertrauen 2018. Media Perspektiven 5/2019, 210-220.

Jowett, Garth S. \& O’Donnell, Victoria (2012). Propaganda and Persuasion. Los Angeles, CA: Sage.

Kirchmann, Kai (2004). Das Gerücht und die Medien. Medientheoretische Annäherungen an einen Sondertypus der informellen Kommunikation. In: Bruhn, M. \& W. Wunderlich (Hrsg.), Medium Gerücht: Studien zu Theorie und Praxis einer kollektiven Kommunikationsform (67-85). Bern: Haupt.

Kohring, Matthias (2006). Öffentlichkeit als Funktionssystem der modernen Gesellschaft. Zur Motivationskraft von Mehrsystemzugehörigkeit. In: Ziemann, A. (Hrsg.), Medien der Gesellschaft - Gesellschaft der Medien (161-181). Konstanz: UVK.

Kohring, Matthias \& Hug, Detlef Matthias (1997). Öffentlichkeit und Journalismus. Medien Journal, 21(1), 15-33. DOI: 10.24989/medienjournal.v21i1.582.

Kohring, Matthias \& Zimmermann, Fabian (2019). Die wissenschaftliche Beobachtung aktueller Desinformation. Eine Entgegnung auf Armin Scholls und Julia Völkers Anmerkungen in »Fake News, aktuelle Desinformationen und das Problem der Systematisierung « in M\&K 2/2019. Medien \& Kommunikationswissenschaft, 67, 319-325. DOI: $10.5771 / 1615-634 X-2019-3-319$

Kuklinski, James H.; Quirk, Paul J.; Jerit, Jennifer, Schwieder, David \& Rich, Robert F. (2000). Misinformation and the Currency of Democratic Citizenship. The Journal of Politics, 62, 790-816. Abgerufen von https://www.jstor.org/stable/2647960. 
Kull, Steven; Ramsay, Call \& Lewis, Evan (2003). Misperceptions, the Media, and the Iraq War. Political Science Quarterly, 118, 569-598. Abgerufen von https://www.jstor.org/stable/30035697.

Lewandowsky, Stephan; Ecker, Ullrich K. H. \& Cook, John (2017). Beyond Misinformation. Understanding and Coping With the »Post-Truth « Era. Journal of Applied Research in Memory and Cognition, 6, 353-369. DOI: 10.1016/j.jarmac.20 17.07.008.

Mahon, James E. (2008). Two definitions of lying. International Journal of Applied Philosophy, 22, 211-230. DOI: 10.5840/ijap200822216.

Mahon, James E. (2015). The Definition of Lying and Deception. The Stanford Encyclopedia of Philosophy (Winter 2016 Edition). Abgerufen von https://plato. stanford.edu/archives/win2016/entries/lying-definition. Abgerufen am 19. September 2019.

Merten, Klaus (1973). Aktualität und Publizität. Zur Kritik der Publizistikwissenschaft. Publizistik, 18, 216-235.

Müller, Jörn (2007). Lüge und Wahrhaftigkeit. Eine philosophische Besichtigung vor dem Hintergrund der Sprechakttheorie. In: Müller, J. \& H.-G. Nissing (Hrsg.), Die Lüge. Ein Alltagsphänomen aus wissenschaftlicher Sicht (27-55). Darmstadt: Wissenschaftliche Buchgesellschaft.

Nelson, Jacob L. \& Taneja, Harsh (2018). The Small, Disloyal Fake News Audience. The Role of Audience Availability in Fake News Consumption. New Media \& Society, 20, 3720-3737. DOI: 10.1177/1461444818758715.

Nyhan, Brendan; Porter, Ethan; Reifler, Jason \& Wood, Thomas J. (2019). Taking Corrections Literally But Not Seriously? The Effects of Information on Factual Beliefs and Candidate Favorability. Political Behavior. Online Vorveröffentlichung. DOI: 10.1007/s11109-019-09528-x

Reedy, Justin; Wells, Chris \& Gastil, John (2014). How Voters Become Misinformed. An Investigation of the Emergence and Consequences of False Factual Beliefs. Social Science Quarterly, 95, 1399-1418. DOI: 10.1111/ssqu.12102.

Reilly, Ian (2012). Satirical Fake News and/as American Political Discourse. The Journal of American Culture, 35, 258-275. DOI: 10.1111/j.1542-734X.2012.00812.x.

Scholl, Armin \& Weischenberg, Siegfried (1998). Journalismus in der Gesellschaft. Theorie, Methodologie und Empirie. Wiesbaden: Westdeutscher Verlag.

Shin, Jieun; Jian, Lian; Driscoll, Kevin \& Bar, François (2018). The Diffusion of Misinformation on Social Media. Temporal Pattern, Message, and Source. Computers in Human Behavior, 83, 278-287. DOI: 10.1016/j.chb.2018.02.008.

Swami, Viren; Barron, David; Weis, Laura \& Furnham, Adrian (2018). To Brexit or Not to Brexit. The Roles of Islamophobia, Conspiracist Beliefs, and Integrated Threat in Voting Intentions for the United Kingdom European Union Membership Referendum. British Journal of Psychology, 109, 156-179. DOI: 10.1111/bjop.12252.

Thorson, Emily (2016). Belief Echoes. The Persistent Effects of Corrected Misinformation. Political Communication, 33, 460-480. DOI: 10.1080/10584609.2015. 1102187. 
Thummes, Kerstin (2013). Täuschung in der strategischen Kommunikation. Eine kommunikationswissenschaftliche Analyse. Wiesbaden: Springer VS.

Vosoughi, Soroush; Roy, Deb \& Aral, Sinan (2018). The Spread of True and False News Online. Science, 359, 1146-1151. DOI: 10.1126/science.aap9559.

Wardle, Claire \& Derakhshan, Hossein (2017). Information Disorder. Toward an Interdisciplinary Framework for Research and Policy Making. Strasburg: Council of Europe. Abgerufen von https://rm.coe.int/information-disorder-toward-an-interdiscipli nary-framework-for-researc/168076277c. Abgerufen am 19. September 2019.

Weeks, Brian E. \& Garrett, R. Kelly (2014). Electoral Consequences of Political Rumors. Motivated Reasoning, Candidate Rumors, and Vote Choice During the 2008 U.S. Presidential Election. International Journal of Public Opinion Research, 26, 401-422. DOI: 10.1093/ijpor/edu005.

Wells, Chris; Reedy, Justin; Gastil, John \& Lee, Carolyn (2009). Information Distortion and Voting Choices. The Origins and Effects of Factual Beliefs in Initiative Elections. Political Psychology, 30, 953-969. DOI: 10.1111/j.1467-9221.2009.00735.x.

Zimmermann, Fabian \& Kohring, Matthias (2018). »Fake News« als aktuelle Desinformation. Systematische Bestimmung eines heterogenen Begriffs. Medien \& Kommunikationswissenschaft, 66, 526-541. DOI: 10.5771/1615-634X-2018-4-526.

Zimmermann, Fabian \& Kohring, Matthias (2020). Mistrust, Disinforming News, and Vote Choice. A Panel Survey on the Origins and Consequences of Believing Disinformation in the 2017 German Parliamentary Election. Political Communication. Online Vorveröffentlichung. DOI: 10.1080/10584609.2019.1686095. 
